\title{
Comparing ICH-leach and Leach Descendents on Image Transfer using DCT
}

\author{
Bennani Taj, Mhamed Ait Kbir \\ Laboratoiry LIST, Faculty of Science and Technology of Tangier, University Abdelmalek Essaadi, Morocco
}

\begin{tabular}{l}
\hline \hline Article Info \\
\hline Article history: \\
Received Dec 22, 2017 \\
Revised Feb 19, 2018 \\
Accepted Feb 26, 2018 \\
\hline
\end{tabular}

\section{Keyword:}

Frequency domain

ICH-leach

Leach protocol

OpenCV

WMSN

\begin{abstract}
The development and miniaturization of CMOS (microphones and cameras) in the last years have allowed the creation of WMSN (Wireless Multimedia Sensor Network). Therefore, transferring multimedia content through the network has become an important field of research. It transmits the recorded multimedia data wirelessly from a node to another to reach the Sink (base station). Thus, routing protocols make a big contribution in this process, because they participate in optimizing the node's resource usage. Since Leach protocol was designed only to minimize energy consumption of the network. The goal of this paper is to compare our protocol in tnansfering images with other Leach protocol descendants. By using the application layer, we applied the jpeg compression using the frequency domain on images before sending them to the network. In this paper, readers will find statistics concerning the lifetime of the network, the energy consumption and most importantly statistics about received images. Also, we used Castalia framework to simulate real conditions of transmission simulation results proved the efficiency of our protocol by prolonging the lifetime of the network and transmitting more images with better quality compared to other protocols.
\end{abstract}

Copyright $\left({ }_{0} 2018\right.$ Institute of Advanced Engineering and Science. All rights reserved.

\section{Corresponding Author:}

Bennani Taj, Laboratoiry LIST,

Faculty of Science and Technology of Tangier, University Abdelmalek Essaadi, Morocco.

Email: Bennani.taj@gmail.com

\section{INTRODUCTION}

The development in micro-electro mechanical system has made possible the miniaturization and the low cost of sensors. Actually, they do multiple tasks like sensing the environment, making computation and communicating wirelessly. A sensor is composed of Multiple components like processor, battery and communicating device. Sensors collect information from the environment (pressure, temperature, etc...), and transform them into electrical signal in order to be treated by the processor unit [1]. After getting the information, the sensors either send them to other sensors or store them. Actually, resources of sensors must be managed efficiently because of multiple limitations such as memory, data processing, power of communication and most importantly energy. In fact, energy is responsible for the lifetime of the sensors. It's essential to take care of this issue [2]. The Figure 1 shows the structure of a sensor.

WSN (Wireless sensor network) is composed of several sensors. Each sensor capture data using scalar sensors, and transfer them to the base station. Actually, many application fields use WSN like agriculture applications [3], underwater applications [4], smart home [5] etc. It is characterized by the following constraints: high bandwidth demand, resource constraints and application-specification [6]. However, routing protocols in WSN consume a big portion of energy. As a result, it affects the lifetime of the network. In fact, there are two kinds of routing protocols: planning protocols and clustered protocols. The first kind considers all the nodes at the same level. Actually, this category includes multiple routing protocols 
such as Multipath routing ring [7], GPSR [8], multipath routing protocol [9] etc. The second one organizes the nodes into groups called clusters. Many routing protocols enter in this category, such like Leach [10], balanced Leach [11], ICH-Leach [12], and M-Leach [13] etc. Nevertheless, some of these protocols could be applied to multimedia transfer.

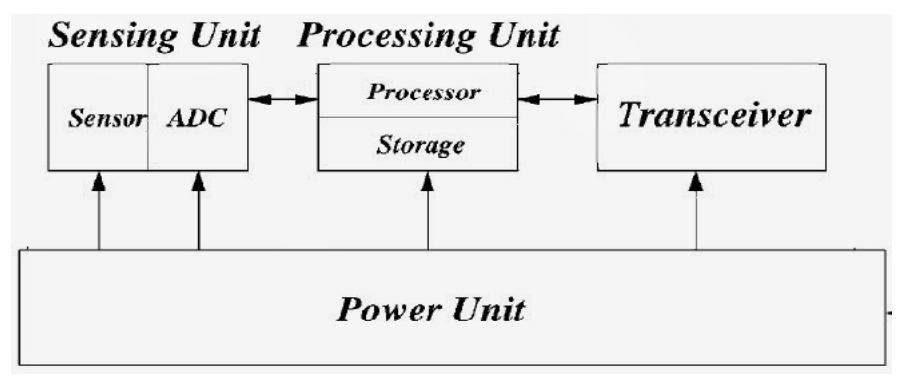

Figure 1. Structure of a sensor

WMSN is formed of sensors that can capture and transmit multimedia content, such as audio, video streams and images. It has multiple architectures that could be used. The first one is the single-tier flat in which sensors are homogeneous and the processing is distributed. The second one is the single-tier clustered which has a heterogeneous nodes and concentrated processing. The last one is multi-tier which has heterogeneous nodes and distributed processing. In order to transmit multimedia data perfectly, the WMSN needs some QoS requirements [14]: the limitation in term of resources, the lack of high band, the use of cross-layer and the coding of multimedia content. Actually, many compression techniques are used during the transmission process.

An Image could be perceived in two ways: frequency or spatial domain. The first one is obtained by applying DCT [15] (the Fourier transform) on spatial domain. In JPEG image coding, we can use different techniques based on DCT. in the spatial domain we consider the image as 2D plane, where pixels has coordinates $(\mathrm{x}, \mathrm{y})$. We used the JPEG coding in our application, which uses a compression based on DCT. Thus, the amount of sent packet is reduced and energy consumption is less [16].

In our work, we use Leach protocol, which is a Low Energy hierarchical protocol. This protocol uses data fusion and rounds (period of time). In each round, sensors, self organized themselves into clusters in which the Cluster-Head $(\mathrm{CH})$ is the active node. In fact, one of the big advantages of Leach is saving energy [10], but cannot be used in WMSN [17]. Actually, many improvements have been made since the original version of Leach. Our improved protocol uses the position of the sink and the position the intermediate $\mathrm{CH}$ in consederation before transmiting. Then, we compared it with many versions of Leach.The results showed that our protocol not only extends the lifetime of the network, but can transmit more images compared to other protocols. In this paper, we are going to see the following chapters. First, we will expose the related works. Then, we will expose our ICH-LEACH. After that, we will outline our application and discuss results obtained using ICH-Leach, MLD-Leach, M-Leach and Leach. Finally, we are going to conclude our work.

\section{RELATED WORKS}

\subsection{Leach protocol}

In 2000, Wendi B. Heinzelman proposed a protocol called LEACH [10] which use a series of equal period of time called rounds. Each round comprises of two steps: cluster setup phase and steady phase. In the first one, nodes decide to be $\mathrm{CH}$ or not for the current round by picking a random value between 0 and 1 . The chosen number will determine if the node is a $\mathrm{CH}$ or not by comparing it with the threshold $\mathrm{T}(\mathrm{n})$. The formula 1 shows how we calculate the threshold.

$$
T(n)=\left\{\begin{array}{c}
\frac{P}{1-P *\left(r *\left(r * \bmod \frac{1}{P}\right)\right.}, n \in G \\
0, \text { else }
\end{array}\right.
$$

$\mathrm{P}$ is the wanted percentage of $\mathrm{CHs}, \mathrm{r}$ is the actual round and $\mathrm{G}$ is the list of nodes that have not been elected $\mathrm{CH}$ in the last $1 / \mathrm{P}$ rounds. When a node elects itself as a $\mathrm{CH}$, It must not be chosen again in the next 
1/P rounds. Each $\mathrm{CH}$ of the current round advertises itself by broadcasting an invitation to all other nodes. When the non $\mathrm{CH}$ nodes receive that invitation, they decide to join the cluster-head which has the strongest signal intensity. By forming the clusters the setup-phase ends. Then, the $\mathrm{CH}$ will assign for every member node a limited period of time of transmission called frames. In each frame, the nodes will be allowed to send their data to the their corresponding $\mathrm{CH}$ using TDMA. In the steady phase, the nodes send their packets to the $\mathrm{CH}$ during their specific frame. The Figure 2 shows this process.

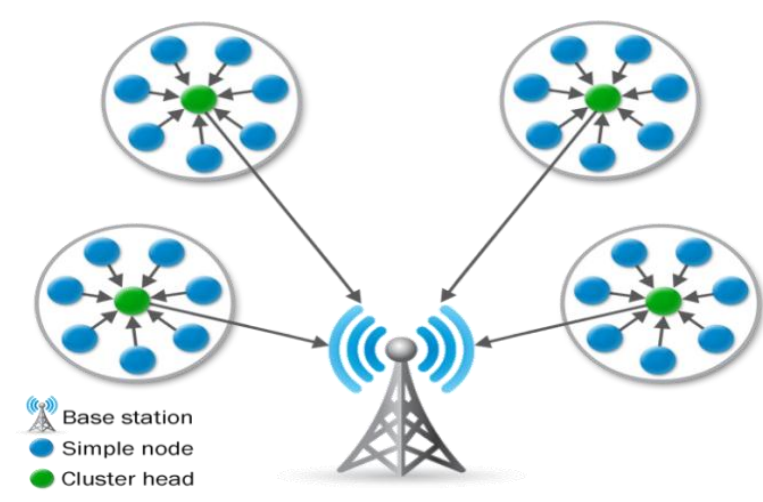

Figure 2. Transmission process in Leach protocol

\subsection{M-LEACH protocol}

Leach has several drawbacks even if it makes energy consumption a priority compared to plane multipath routing protocols. First, the cluster-heads communicate directly to the sink which causes congestion and high energy consumption of transmission. Then, the cluster heads that are far from the sink will not be capable to reach the sink. For this reason, multiple improvements of leach were developed to overcome these drawbacks. M-leach is one of these protocols. It uses multipath to transmit data by making cluster-heads intermediate nodes to reach the sink. The intermediate $\mathrm{CHs}$ are chosen following the shortest path to the base station.

\subsection{MLD-LEACH protocol}

MLD-Leach [18] is a protocol that uses the notion of layers that start from the sink. After the formation, cluster-heads of upper layers have a periodic update of delays with cluster-heads of lower layers as shown in Figure 3. In transmission phase, the cluster-heads of each layer, choose the cluster-head of lower layer which has the minimum delay then the minimum distance.

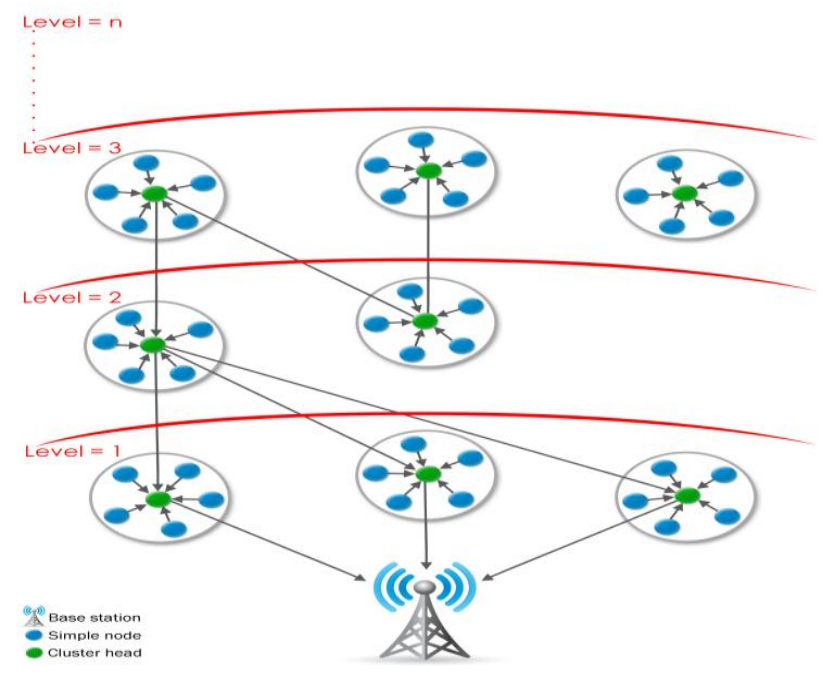

Figure 3. Multilayer structure 


\section{ICH-LEACH PROTOCOL}

ICH-Leach [12] is an improved Leach protocol that extends the lifetime of nodes by reducing the energy consumption and sends more images to the base station. Our protocol follows the same steps of Leach, and uses the same percentage of $\mathrm{CH}$. To send more images from a $\mathrm{CH}$ to the base station in one hop, data transmission needs high energy consumption [17]. Furthermore, if a $\mathrm{CH}$ can't reach the sink the data is lost. Thus, by using $\mathrm{CH}$ as intermediate node the consumed energy is reduced, and the number of sent images is high even if the distance is far from the base station.

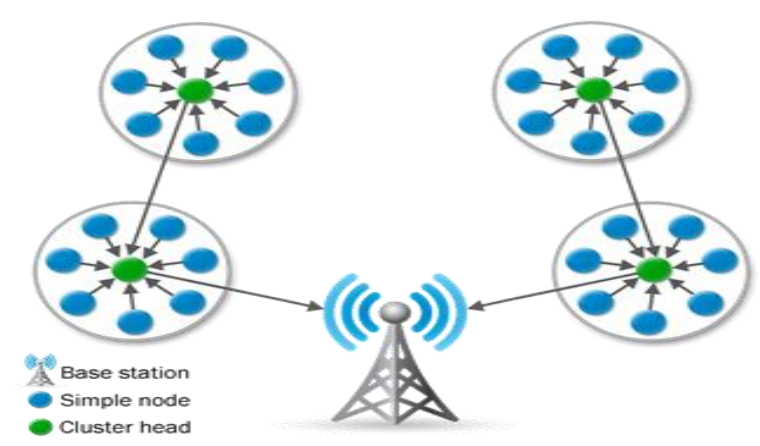

Figure 4. ICH-LEACH transmission process

The intermediate $\mathrm{CH}$ in the Figure 4 is picked based on the position of the sink, position of $\mathrm{CH}$ and the position of the intermediate $\mathrm{CH}$. Thus, a $\mathrm{CH}$ can reach the sink no matter the distance.

\subsection{Initialization phase}

At first, the base station advertises its position by diffusing a packet that contains its position to the network using the maximum transmission of energy. When a sensor receives the packets, it saves the position of the sink and the signal strength of the received packet. Then, the node starts the election process. If it elects itself as a $\mathrm{CH}$, it retransmit the packet by adding its coordinates. Thus, the formation of clusters begins. The stored position of the sink will help the $\mathrm{CH}$ to decide whether or not to send data directly or not.

\subsection{Cluster auto-organisation}

The Figure 5 shows the cluster formation of our protocol.

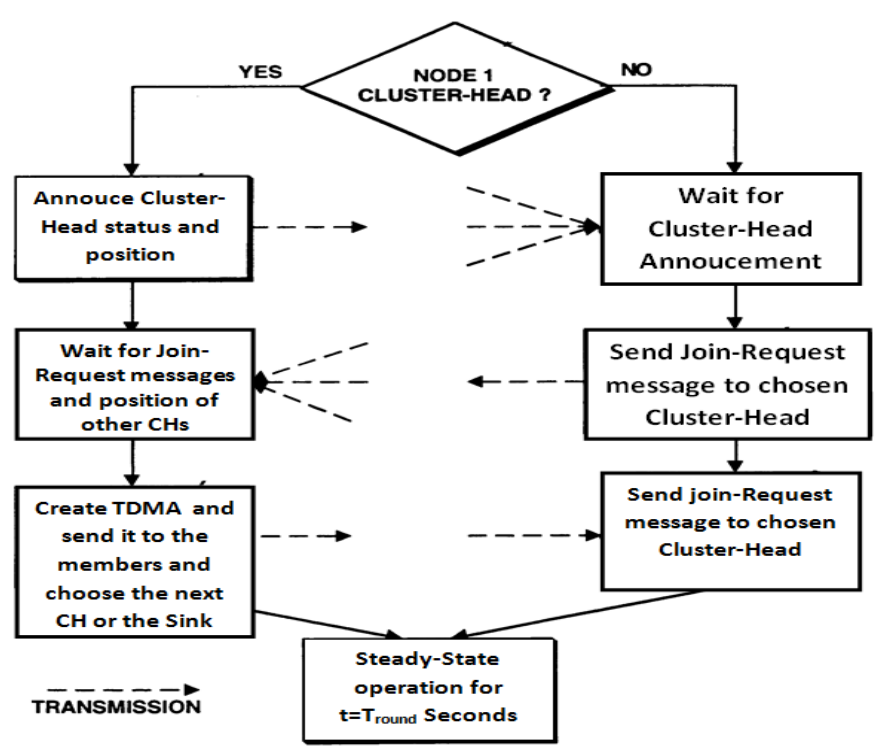

Figure 5. ICH-LEACH cluster formation process 


\subsection{Transmission process}

In this phase, the $\mathrm{CHs}$ transmit their data to the base station if it is the closest node. However, it sends them to the intermediate cluster-head if the sink is not reachable. If a $\mathrm{CH}$ receives a packet, it saves it until its turn comes.

\section{SIMULATIONS AND RESULTS}

\subsection{Application layer}

In our application, we treat the sensed images before sending them through to the network by compressing them. Therefore, we created an application in the application layer to make this process by using the frequency domain of images to make our transformation. In transmission phase, we take an image that is divided into small blocks of $8 \times 8$. First, each block is divided into multiple planes which present a color. Second, we process each plane using DCT. Third, we apply the quantization process on the DCT matrix. Fourth, we use the RLE compression technique to minimize the size of packets before transmission. Finally, we send the matrix of frequency values to network with its meta-data. Once the sink receives the data, we create the quantization matrix by applying the reverse RLE. Then, we get the DCT matrix by applying the reverse quantization process. After that, we create the frequency values of each plan, and apply the reverse DCT on each plane. Finally, by using the metadata we construct the image progressively. The Figure 6 resumes this process. In our work, we used a gray image.

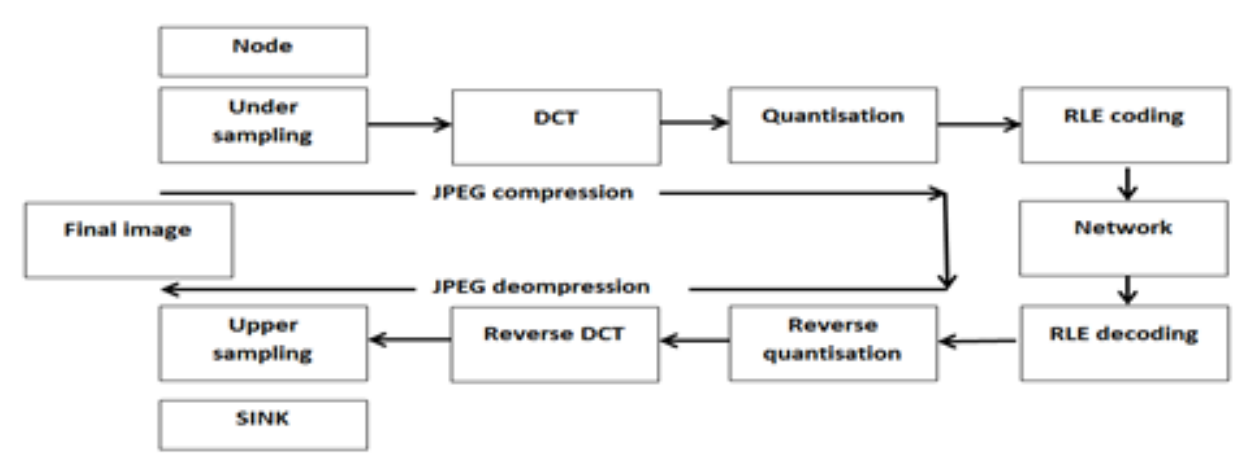

Figure 6. Compression and decompression process of images

\subsection{Simulation parameters}

In this paragraph, we will detail the simulation parameters and the results that we obtained. We used the framework Castalia [19] to make our simulations, and used the radio model CC2240 [20] to replace a real radio transmission. In fact, the number of rounds is too small because of the radio and the effect of round length on multimedia transfer [17]. Also, we compared ICH-Leach with the original leach, M-leach and MLD-Leach.

Table 1. Simulation Parameters

\begin{tabular}{cc}
\hline Parameter & Value \\
\hline Topology size & $100 \times 100 \mathrm{~m} 2$ \\
Number of nodes & 100 \\
CH probability & 0.05 \\
Number of trials & 20 \\
Initial power & $30 \mathrm{j}$ \\
BS position & $(0,0)$ \\
Size of image & $64 \times 64$ \\
Number of sent images & 2 \\
Delay between images & $300 \mathrm{~s}$ \\
Delay between sending packets & $1 \mathrm{~s}$ \\
Quantization percentage & $85 \%$ \\
\hline
\end{tabular}

In our simulation we suppose that the sink can't reach all the nodes. The table 1 shows the proprieties that we used in our simulation. 


\subsection{Analysis of results}

In this paragraph, we intend to evaluate our protocol using two approaches. The first approach is the duration of the network and energy consumption per rounds. In fact, this parameter shows us how a routing protocol is efficient in term of extending the lifetime of nodes that form the network and energy consumption. In The multimedia approach, we are going to use different metrics. The first one is measuring the number of images per quality. Actually, this metric allows us to measure the quality of received images. The second one is measuring the lost packets during the transmission. It shows us how the network reacts in term of delivering packets, which has an influence on the number of received images and their quality.

The Figure 7 shows the energy that is consumed by the entire network. It shows clearly that leach protocol has the highest energy consumption among the four. Also, that Leach, M-leach and MLD-Leach consume less than Leach. Nevertheless, ICH-leach consumes lower energy compared to other protocols. These results could be explained by the fact that Leach uses a full energy transmission to reach the sink. Thus, the energy consumption per round is high, compared to other protocols. M-Leach and MLD-Leach use multiple hops, which helps to minimize energy consumption per round of the network compared to Leach. Finally, ICH-Leach uses at maximum two hopes based on the position of the base station and the node source. Consequently, the energy consumed by the network is reduced to the minimum.

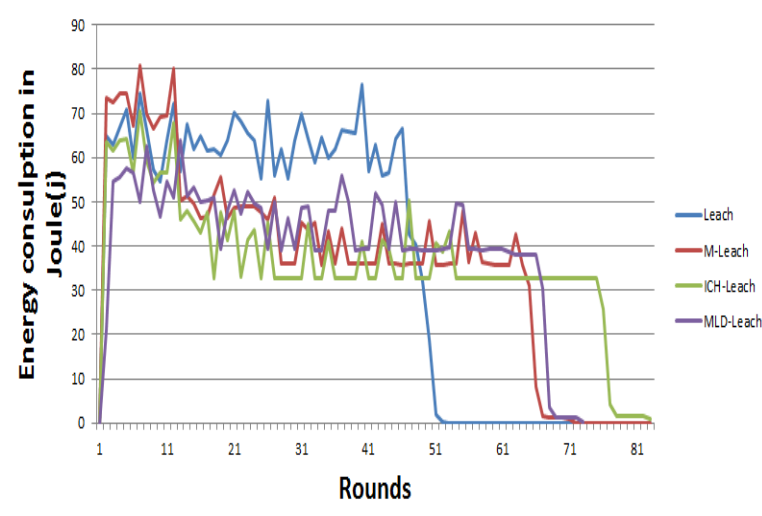

Figure 7. Energy consumption per round

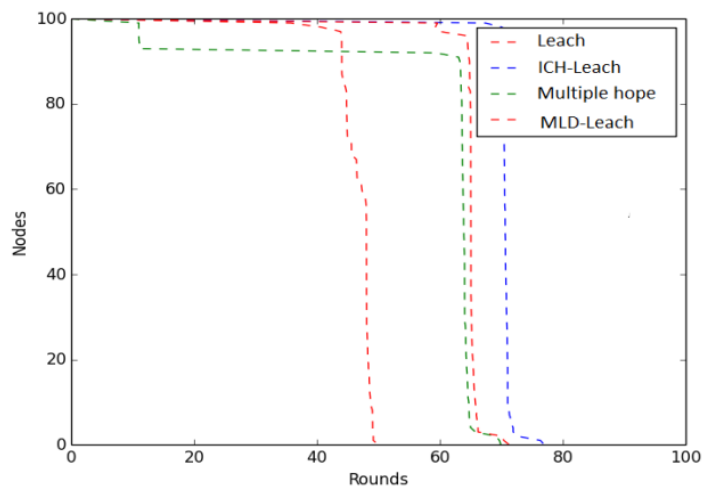

Figure 8. Dead node per protocol

In the original protocol, the high energy consumption per round due to the full energy transmission causes the short duration of the network comparing to the other ones. On the other side, M-leach has a longer lifetime of the network compared to Leach. Although, the multi-hop in M-leach uses intermediate clusterheads to reach the sink based only on distance, which could over-uses some cluster-heads. Hence, it has a direct effect on these $\mathrm{CHs}$ by dropping their energy faster than others as depicted in Figure 10. Thus, some nodes die in the first rounds. The MLD-leach has a longer lifetime of the network compared to M-Leach, even if both of them use multi-hop. This result can be justified by the fact that the amount of intermediate $\mathrm{CHs}$ depends on the number of layers from the sink. Finally, the ICH-Leach proves more consistency and extends the duration of the network compared to other protocols, and can send data to the sink on one hope if it is the nearest. Otherwise, the $\mathrm{CH}$ chooses the nearest $\mathrm{CH}$ As an intermediate $\mathrm{CH}$. Also, the power of transmission is adjusted according to next node. This policy has two benefits. First, reducing the congestion of the network. Second, economizing energy, which is confirmed by the obtained results in Figure 7 concerning energy consumption of all nodes. Figure 8 shows the dead node per protocol.

The Figures 9 shows that most of the constructed image by the sink using Leach is less than $34 \%$ of the quality of the original image. Also, it has a high level of lost packet. In Figure 10, the M-leach can transmits a better quality $(100 \%)$ in big numbers and the rest of received images are less than $64 \%$. In the Figure 11, MLD-Leach has big numbers of completed images with a big number of received images. In Figure 12, the ICH-Leach has the biggest number of received packets with the highest quality. Also more than $80 \%$ of received images are $100 \%$ of the original image, and the rest is less than $64 \%$. The Figure 13 shows that ICH-Leach has the minimum number of lost packets and big number of images with high quality.

We can explain these results for many reasons. Concerning Leach results can be explained by the congestion, which is caused by the single hop from CHs. For M-Leach results can be explained the big number of lost packets because of the high amount of intermediates CHs. For MLD-leach, results can be explained by the fix number of intermediate nodes with the number of layers and varying the next node depending on the network. Thus, we have less congestion and less loss packets. Finally, results of ICH-Leach 
Can be explained by the fact that it uses one intermediate node at most. So, less congestion around the sink and less packets in the buffer.

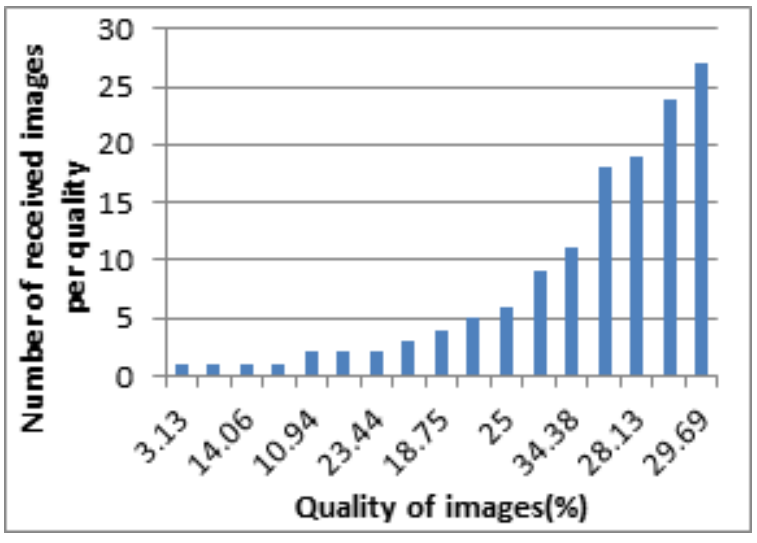

Figure 9. Number of images per quality using LEACH protocol

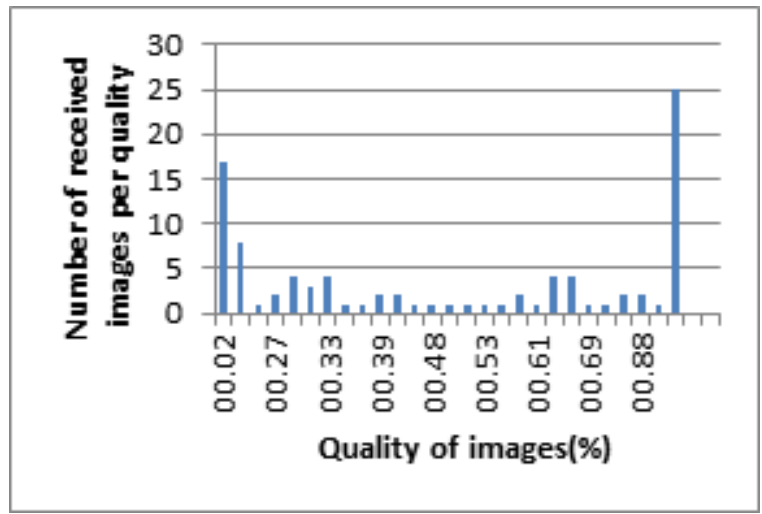

Figure 11. Number of images per quality using MLD-LEACH protocol

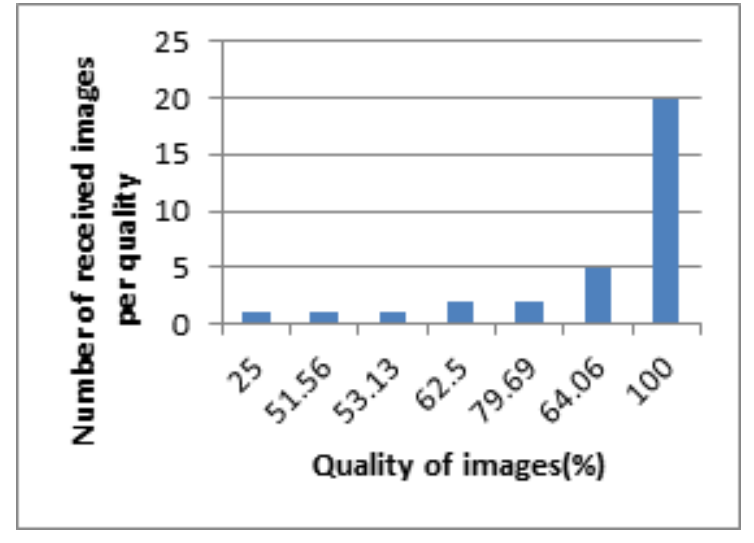

Figure 10. Number of images per quality using M-LEACH protocol

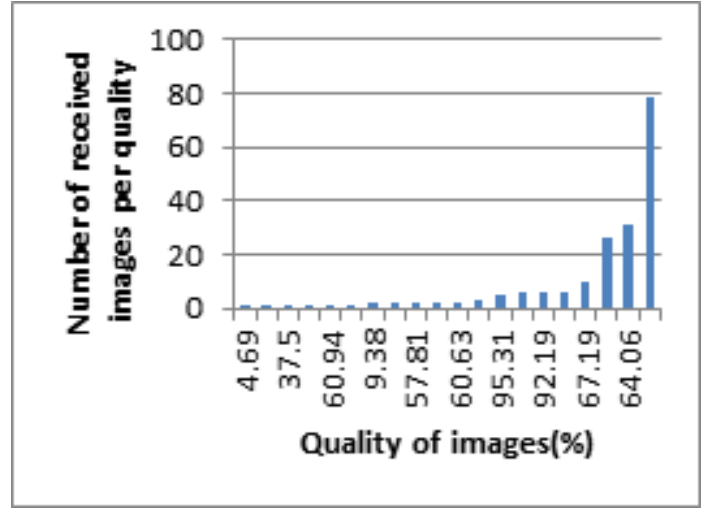

Figure 12. Number of images per quality using ICH-Leach protocol

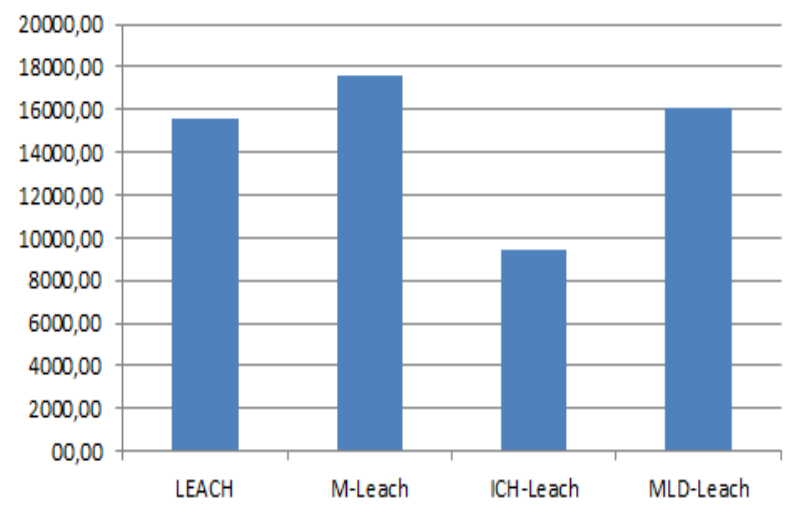

Figure 13. Number of lost packets per protocol 


\section{CONCLUSION}

During this work, we used the DCT compression technique on images, which uses the frequency domain to reduce the number of packets during transmission process. Then, we used Leach, M-leach, MLDLeach and ICH-Leach as routing protocols to convey the images to the sink to compare them. As a result, ICH-Leach increases the quality and the number of transferred images. In addition, it reduces energy consumption. Since MLD-Leach protocol is designed to manage energy consumption by using layers and taking distance into account, our protocol shows good performances in term of energy consumption and lifetime of the network. Also, it fixes the issue of M-Leach, which is the big number of intermediate nodes. This problem creates a big waiting list of packets. Thus, our protocol is more suitable for transferring multimedia content. As a perspective, we want to develop a routing protocol that adapts the quality of transmitted images in function of the state of the network.

\section{REFERENCES}

[1] D. Estrin, D. Culler, K. Pister, and G. Sukhatme, "Connecting the physical world with pervasive networks," IEEE Pervasive Comput, vol. 1, no. 1, pp. 59-69, Jan 2002.

[2] K. C. Rahman, "A survey on sensor network," Journal of Computer and Information Technology, vol. 1, no 1, pp. 76-87, 2010

[3] S. Navulur, S. Navulur, C. S. S. As, et G. P. Mn, "Agricultural Management through Wireless Sensors and Internet of Things", International Journal of Electrical and Computer Engineering (IJECE), vol. 7, no 6, p. 3492, Dec, 2017.

[4] P. V. Amoli, "An Overview on Current Researches on Underwater Sensor Networks: Applications, Challenges and Future Trends", International Journal of Electrical and Computer Engineering (IJECE), vol. 6, no. 3, p. 955, 2016.

[5] H. Sayuti et al., "Smart Home and Ambient Assisted Living Based on the Internet of Things", International Journal of Electrical and Computer Engineering (IJECE), vol. 7, no 3, p. 1480, 2017.

[6] J. Yick, B. Mukherjee, et D. Ghosal, "Wireless sensor network survey", Computer Networks, vol. 52, no 12, pp. 2292-2330, 2008.

[7] A. Alanazi et K. Elleithy, "Real-Time QoS Routing Protocols in Wireless Multimedia Sensor Networks: Study and Analysis", Sensors, vol. 15, no 9, pp. 22209-22233, Sept. 2015.

[8] Y. Sun, J. Guo, et Y. Yao, "Speed Up-Greedy Perimeter Stateless Routing Protocol for Wireless Sensor Networks (SU-GPSR)", in High Performance Switching and Routing (HPSR), 2017 IEEE 18th International Conference on, 2017, pp. 1-6.

[9] S. Kim, H. Cho, T. Yang, C. Kim, et S.-H. Kim, "Low-Cost Multipath Routing Protocol by Adapting Opportunistic Routing in Wireless Sensor Networks", in Wireless Communications and Networking Conference (WCNC), 2017 IEEE, 2017, pp. 1-6.

[10] W. R. Heinzelman, A. Chandrakasan, and H. Balakrishnan, "Energy-efficient communication protocol for wireless microsensor networks," in Proceedings of the 33rd Annual Hawaii International Conference on System Sciences, vol. 2, no. 10, 2000.

[11] W. Wang et Y. Peng, "LEACH algorithm based on Load balancing", Indonesian Journal of Electrical Engineering and Computer Science, vol. 11, no. 9, pp. 5329-5335, 2013.

[12] M. B. M. Taj and M. A. Kbir, "ICH-LEACH: An enhanced LEACH protocol for wireless sensor network", in 2016 International Conference on Advanced Communication Systems and Information Security (ACOSIS), pp. 1-5,2016.

[13] A. Muntasa, I. A. Sirajudin, M. H. Purnomo, "Appearance global and local structure fusion for face image recognition”, TELKOMNIKA (Telecommunication, Computing, Electronics and Control), 2011, vol. 9, no. 1, pp.125-132.

[14] M. Abazeed, N. Faisal, S. Zubair, and A. Ali, "Routing Protocols for Wireless Multimedia Sensor Network: A Survey", Journal of Sensors, vol. 2013, pp. 1-11.

[15] Li Ze-Nian, Drew Mark S. ch8. lossy image compression techniques in Fundamentals of multimedia. Prentice-Hall, 2004. 0130618721.

[16] A. Mammeri, A. Khoumsi, D. Ziou, and B. Hadjou,"Modeling and adapting JPEG to the energy requirements of VSN," in Computer Communications and Networks, ICCCN'08. Proceedings of 17th International Conference on 2008, pp. 1-6, 2008.

[17] M. A. Kbir and M. Bennani, "Round time adaptation for image transfering over wireless sensor networks leach protocol," in 2016 4th IEEE International Colloquium on Information Science and Technology (CiSt), pp. 896-900, 2016.

[18] M. Bennani et M. Kbir, "MLD-LEACH: An Enhanced Leach Protocol for Multimedia Wireless Sensor Network", Journal of Theoretical \& Applied Information Technology, vol. 95, no. 10, 2017.

[19] "Home." [Online]. Available: https://castalia.forge.nicta.com.au/index.php/en/. (Accessed on 21-Sep-2016).

[20] "CC2420 | Proprietary $2.4 \mathrm{GHz} \mid$ Wireless Connectivity | Description \& parametrics." [Online]. Available: http://www.ti.com/product/CC2420. (Accessed on 29-Sep-2016). 